\title{
Worsening of unrecognized tumour-induced osteomalacia with inadvertent use of recombinant human parathyroid hormone
}

\author{
Rimesh Pal', Kanhaiya Agrawal', Setu Gupta ${ }^{2}$, Anil Bhansali', Arunanshu Behera ${ }^{3}$, Sanjay Kumar Bhadada ${ }^{1}$ \\ ${ }^{1}$ Department of Endocrinology, Postgraduate Institute of Medical Education and Research, Chandigarh, India \\ ${ }^{2}$ Department of Internal Medicine, Postgraduate Institute of Medical Education and Research, Chandigarh, India \\ ${ }^{3}$ Department of General Surgery, Postgraduate Institute of Medical Education and Research, Chandigarh, India
}

Key words: tumour-induced osteomalacia; teriparatide; hypophosphataemia; fragility fracture

\section{Case presentation}

A 45-year-old man presented with lower limb proximal muscle weakness that had begun 1.5 years back. He developed bilateral fracture neck femora following a trivial fall onto the ground one year ago (Fig. 1A). He underwent bilateral hip replacement for the same at a private healthcare facility. He was discharged on calcium and vitamin D supplements along with recombinant human parathyroid hormone (rhPTH/teriparatide) at a dose of 20 micrograms once daily to promote fracture healing. After one month of teriparatide therapy, his symptoms aggravated. His lower limb muscle power declined, soon requiring support to stand up and walk. This was associated with marked bony pain over the lower limbs. Six months later he became bedbound. The patient stopped taking teriparatide and subsequently presented to our institute. A review of previous investigations (performed at a private hospital) revealed serum calcium $9.2 \mathrm{mg} / \mathrm{dL}$ (range: 8.6-10.4), total alkaline phosphatase (ALP) 672 IU/L (range:74-138), and 25-hydroxyvitamin D 18 $\mathrm{ng} / \mathrm{mL}$. However, a serum phosphorous report was not available. Histopathology of the excised femoral heads was reported as having osteomalacia.

Physical examination revealed lower limb proximal myopathy. Distal muscle power, deep tendon reflexes and sensory system were intact. Biochemical investigations revealed hypophosphataemia $(0.9-1.1 \mathrm{mg} / \mathrm{dL}$, range: 2.8-4.5), elevated ALP (790 IU/L, range: 78-128), and vitamin D sufficiency. Urinalysis revealed renal phosphate wasting with low tubular reabsorption of phosphate corrected for glomerular filtration rate (TmP/GFR $=0.409 \mathrm{mg} / \mathrm{dL}$, age-specific range: $2.8-4.2$ ).
Serum FGF23 level (measured by C-terminal ELISA, Quidel, Immutopics) was elevated at 513.8 RU/mL (range: 0.0-150.0) with inappropriately low 1, 25-dihydroxyvitamin D (24.2 pg/mL, range: 19.9-79.3). Somatostatin-receptor (SSTR)-based scintigraphy with ${ }^{68} \mathrm{Ga}$-DOTATATE PET-CT showed a $1 \mathrm{~cm} \times 1 \mathrm{~cm}$ tracer-avid soft tissue lesion in the medial aspect of the left thigh (Fig. 1B). It was concordant on ultrasonography. The lesion was excised with a wide resection margin. Serum phosphate levels rose, reaching $2.8 \mathrm{mg} / \mathrm{dL}$ on the fourth post-operative day. Simultaneously, serum FGF23 levels fell to $103.8 \mathrm{RU} / \mathrm{mL}$. Histopathology of the excised lesion was suggestive of phosphaturic mesenchymal tumour. At two months follow-up, there has been a marked symptomatic improvement with the patient able to stand and walk on his own. His serum phosphate has increased to $3.8 \mathrm{mg} / \mathrm{dL}$ and his ALP level has come down to $288 \mathrm{IU} / \mathrm{L}$.

\section{Discussion}

Tumour-induced osteomalacia is a rare paraneoplastic syndrome characterised by refractory hypophosphataemia and is usually associated with benign mesenchymal soft-tissue tumours that produce FGF23 [1, 2]. FGF23, acting via FGF receptor 1 (FGFR1) and co-receptor $\alpha$-klotho on renal proximal tubules promotes internalisation of sodium-phosphate co-transporters (NaPi) from the luminal membrane to the cytoplasm, thereby reducing tubular phosphate reabsorption and causing phosphaturia [3]. In addition, FGF23 also reduces renal mRNA and protein levels of NaPi-2a co-transporters [4]. Similarly, PTH, acting via G-protein-coupled PTH

Prof. Dr. Sanjay Kumar Bhadada, Department of Endocrinology, Postgraduate Institute of Medical Education and Research,

Chandigarh 160012, tel: (+91) 98766024 48; e-mail: bhadadask@rediffmail.com 


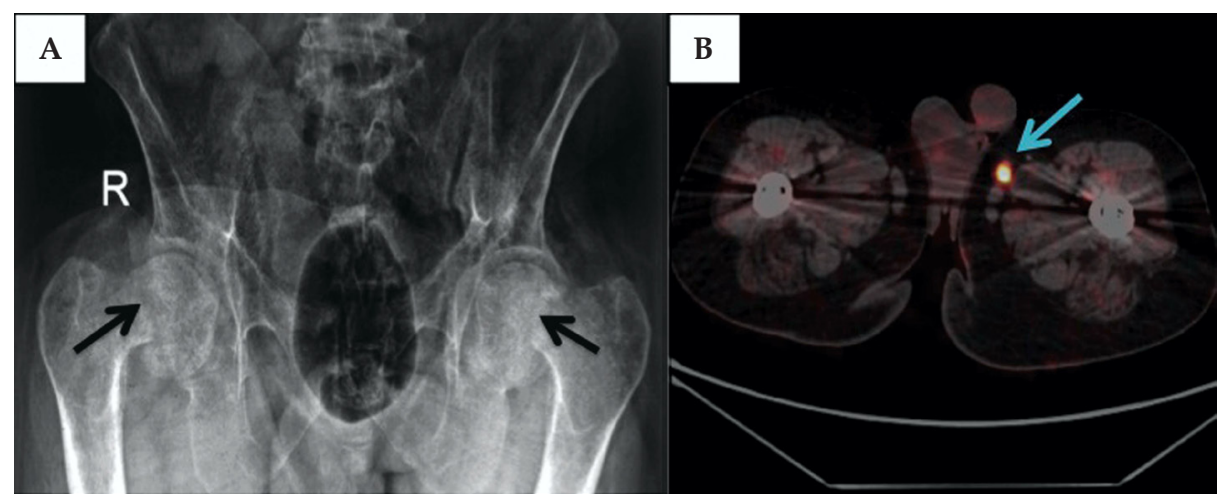

Figure 1.A. Antero-posterior radiograph of the pelvis showing bilateral fracture neck femora (marked in black arrows). B. Fused ${ }^{68} \mathrm{G} a$-DOTATATE PET-CT image showing a $1 \mathrm{~cm} \times 1 \mathrm{~cm}$ tracer-avid soft tissue lesion (marked in blue arrow) in the subcutaneous plane of the medial aspect of the left thigh region just anterior to the belly of the gracilis muscle [maximum standardised uptake value (SUVmax) of 12.8]

receptor 1 (PTHR1), increases cAMP in proximal tubular cells, reducing luminal expression of $\mathrm{NaPi}$, culminating in phosphaturia. In fact, the internalisation of $\mathrm{NaPi}$ co-transporters seems to be faster and more effective under the effect of PTH as compared to FGF23 [4, 5]. In addition, PTH, either directly or via increase in serum 1,25-dihydroxyvitamin $D$, tends to increase FGF23 secretion from bone cells [5,6]. Thus, an excess of endogenous circulating PTH is expected to increase the serum levels of FGF23 as well as potentiate its action at the renal level. The same should hold true with exogenously administered hPTH as well. Studies have shown that infusion of hPTH (1-34) in healthy human volunteers leads to a rise in circulating FGF23 levels, mostly mediated by a rise a serum 1,25-dihydroxyvitamin D levels. Thus, a milieu, wherein PTH and FGF23 are both in excess will aggravate hypophosphataemia. This phenomenon is exemplified in our patient where the hypophosphataemic symptoms were exacerbated on inadvertently adding rhPTH in a state of underlying FGF23 excess.

Tumour-induced osteomalacia must have been the underlying cause of the fragility fractures involving the neck of the femora; however, an attempt to promote fracture healing with teriparatide acted as fuel to the fire, aggravating hypophosphataemia. Although transient hypercalcaemia is a known adverse effect of teriparatide therapy, hypophosphataemia is extremely rare [7]. Refractory hypophosphataemia in a patient on teriparatide should make the clinician think of TIO. The serum phosphate level of the index patient was not assessed before initiating teriparatide. With widespread availability of teriparatide and innumerable biosimilars on the market, there is a temptation among unwary physicians and surgeons to prescribe rhPTH for indications that are not approved by conventional guidelines. Such a practice should be condemned in routine clinical practice. In addition, physicians plan- ning to prescribe teriparatide should beforehand order a basic biochemical panel that must include a minimum of serum calcium, phosphorous, ALP, and creatinine and refrain from its use in patients with unexplained hypophosphataemia.

\section{Conflicts of interest}

The authors declare that they have no conflicts of interest.

\section{Funding sources}

None.

\section{Patient consent}

Informed, written consent was obtained from the patient.

\section{Acknowledgements}

None.

\section{References}

1. Liu S, Quarles LD. How fibroblast growth factor 23 works. J Am Soc Nephrol. 2007; 18(6): 1637-1647, doi: 10.1681/ASN.2007010068, indexed in Pubmed: 17494882

2. Pal R, Bhadada SK, Shingare A, et al. Tumor-induced osteomalacia: experience from three tertiary care centres In India. Endocr Connect. 2019 [Epub ahead of print], doi: 10.1530/EC-18-0552, indexed in Pubmed: 30726771.

3. Minisola S, Peacock M, Fukumoto S, et al. Tumour-induced osteomalacia Nat Rev Dis Primers. 2017; 3: 17044, doi: 10.1038/nrdp.2017.44, indexed in Pubmed: 28703220.

4. Shimada T, Hasegawa H, Yamazaki Y, et al. FGF-23 is a potent regulator of vitamin D metabolism and phosphate homeostasis. J Bone Miner Res. 2004; 19(3): 429-435, doi: 10.1359/JBMR.0301264, indexed in Pubmed: 15040831.

5. Blau JE, Collins MT. The PTH-Vitamin D-FGF23 axis. Rev Endocr Metab Disord. 2015; 16(2): 165-174, doi: 10.1007/s11154-015-9318-z, indexed in Pubmed: 26296372.

6. Lanske B, Razzaque MS. Molecular interactions of FGF23 and PTH in phosphate regulation. Kidney Int. 2014; 86(6): 1072-1074, doi: 10.1038/ki.2014.316, indexed in Pubmed: 25427080.

7. Hajime M, Okada $\mathrm{Y}$, Mori $\mathrm{H}$, et al. A case of teriparatide-induced severe hypophosphatemia and hypercalcemia. J Bone Miner Metab. 2014; 32(5): 601-604, doi: 10.1007/s00774-014-0564-z, indexed in Pubmed: 24553859 . 